\title{
A Scalable Macromodeling Methodology for the Efficient Design of Microwave Filters
}

\author{
Matthias Caenepeel ${ }^{1}$, Krishnan Chemmangat ${ }^{2}$, Francesco Ferranti ${ }^{1}$, Yves Rolain ${ }^{1}$, \\ Tom Dhaene ${ }^{2}$ and Luc Knockaert ${ }^{2}$ \\ ${ }^{1}$ ELEC, Vrije Universiteit Brussel (VUB), Pleinlaan 2, 1050 Elsene, Belgium \\ ${ }^{2}$ Department of Information Technology, Ghent University-iMinds, Gaston \\ Crommenlaan 8 Bus 201, 9050 Gent, Belgium
}

June 10, 2015

\begin{abstract}
The complexity of the design of state-of-the art microwave filters increases steadily over the years. General design techniques available in literature yield relatively good initial designs, but electromagnetic (EM) optimization is often needed to meet the desired specifications. Although interesting optimization strategies exist, they depend on computationally expensive EM simulations. This makes the optimization process time consuming. Moreover, brute force optimization does not provide physical insights in the design and it is only applicable to one set of specifications. If the specifications change, the design and optimization process must be redone. We propose to use a scalable macromodel-based design approach to overcome this. Scalable macromodels can be generated in an efficient and automated way.

So far the inclusion of scalable macromodels in the design cycle of microwave filters
\end{abstract}


has not been studied and discussed. In this paper, we show that scalable macromodels can be included in the design cycle of microwave filters and re-used in multiple design scenarios at a low computational cost. We give guidelines to properly generate and use scalable macromodels in a filter design context. We illustrate the approach on a stateof-the-art design example: a microstrip dual-band bandpass filter with closely spaced pass bands and a complex geometrical structure. The results confirm that scalable macromodels are proper design tools and an efficient and accurate alternative to a computationally expensive EM simulator-based design flow.

\section{Introduction}

The design of microwave filters remains important for both research and applications. Several methods have been proposed to address the design of distributed filters [1,2]. General design techniques yield relatively good initial designs [3]. As the design process does not take into account the non-ideality of the components (such as unwanted couplings between lines or steps in the width of lines), most designs require post-processing fine-tuning to meet the desired specifications. This process involves numerical optimization based on multiple accurate electromagnetic (EM) simulations. Accuracy comes at a price however, these solvers are known to be computationally expensive and hence time consuming. Moreover, EM optimization does not provide the designer with any insights about the influence of the design parameters on the filter response. Even though this results in accurate designs, the process is typically time consuming. Also, the EM optimization remains only applicable to a single set of specifications and if the specifications change this process must be re-done.

To speed up the design process and gain insights about the filter behavior, the EM solver can be replaced by a computationally efficient scalable frequency response model. In this paper, scalable (or parametric) macromodels are used as a compromise between model accuracy and complexity. Generating scalable macromodels to represent the parameterized 
response of microwave systems with respect to frequency and additional design parameters such as geometrical variables and material properties is an active field of research [4-16]. The two main advantages of using scalable macromodels in the design process are:

1. The scalable macromodels replace the expensive EM solver to evaluate the filter response as a function of frequency and the design parameters of interest (e.g. geometrical parameters) over certain ranges. Therefore, these scalable macromodels can be used in different optimization scenarios where changes in the specifications of the filter (e.g. the bandwidth of interest, the selectivity, etc.), need to be examined.

2. The scalable macromodels can also be used to speed up other computationally expensive design activities, such as design exploration and design variability analysis. Design space exploration leads to an understanding of the filter behavior with respect to design parameters without being restricted to design optimization. Design variability analysis enables the evaluation of the system reliability. Since these models are quite cheap to evaluate and also accurate to properly capture the effects of design parameters variations on the filter frequency response, they allow efficiently performing these design tasks and gaining physical insight into the behavior of filters at a low computational cost.

Even though the extraction process of scalable macromodels can be automated using sequential sampling approaches [17], it still requires the designer to specify additional information. For example, the designer must select ranges for the design parameters, for which the model is built. The models hence must be extracted during the design process itself.

In this paper, we show how to include scalable macromodels in the design cycle of microwave filters. We give guidelines to properly generate and use scalable macromodels 
in a filter design context. The focus of this paper is on the practical use of scalable macromodels for design purposes.

We show that scalable macromodels are not only useful for a single design optimization, but they can be re-used to optimize the design to meet multiple sets of specifications. This distinguishes the proposed method from existing model-based optimization methods [18-22] that aim at optimizing a particular performance measure, which leads to restart the modeling step for optimization each time the specifications are changed.

This paper aims to show how scalable macromodels can be effectively and practically used by designers to speed-up the design flow, while achieving accurate results.

We have chosen a state-of-the-art design example of a microstrip dual-band bandpass filter described in [23] to illustrate our approach. In [23], a design method is presented to adjust the center frequency, bandwidth, the position of the transmission zeros and desired ratio of the resonance frequency of the two passbands. This filter consists of two coupled unequal length shunted-line stepped impedance resonators. This example nicely illustrates the proposed macromodel-based design approach, since all the design parameters affect each other and optimization of the design is therefore necessary.

Section 2 describes a state-of-the-art scalable macromodeling method which is coupled with a sequential sampling algorithm for an automated model generation. Section 3 describes the design process and explains how the scalable macromodel can be included in this process. Section 4 validates the proposed macromodel-based design approach with a state-of-the-art filter example. Section 5 discusses limitations of the proposed approach when the number of design paramaters increases and future work. Section 6 summarizes the conclusions. 


\section{Scalable Macromodels for Microwave Filters}

This section introduces a state-of-the-art scalable macromodeling technique that is coupled with a sequential sampling algorithm to obtain an automated model generation framework.

\subsection{Building a scalable macromodel from data samples generated by EM solvers}

The first step of the scalable macromodeling process is to generate a set of multivariate data samples $\left\{\left(s_{n}, \vec{g}_{\boldsymbol{k}}\right), \boldsymbol{H}\left(s_{n}, \overrightarrow{\boldsymbol{g}}_{\boldsymbol{k}}\right)\right\}, n=1, \ldots, N_{s}, k=1, \ldots, K^{\text {tot }}$ that represents a set of parameter-dependent frequency-domain responses. This set depends on the complex frequency $s=j \omega=j 2 \pi f r e q$ and additional $N$ design variables $\vec{g}=\left(g^{(1)}, \ldots, g^{(N)}\right)$. The space that contains the parameters $\vec{g}$ is called design space. For filter structures, these design variables can describe the geometry of the system that a designer vary during the design. These data samples are used to generate a scalable macromodel that is able to efficiently and accurately describe the parameterized behavior of the system under study. The data samples are divided into two datasets: an estimation set and a validation set. The estimation set is utilized to build a scalable macromodel and the validation set is used to validate its modeling capability in design space points not used for the model generation. An efficient sampling algorithm [17] is used to gather data samples located at maximally informative design space positions, i.e. spots in the design space where the response changes rapidly, and then to minimize as much as possible the amount of data samples needed to build and validate a scalable macromodel. This algorithm is briefly described in Section 2.2. In [17], the sequential sampling algorithm is coupled with the scalable macromodeling methods $[15,16]$ to provide an automated modeling process. These

modeling methods $[15,16]$ are based on the use of interpolation of transfer functions and scaling coefficients. Recently, a scalable macromodeling approach has been proposed in 
[24] to enhance the modeling capability of $[15,16]$ by using multiple frequency scaling coefficients. In this paper, we use the scalable macromodeling technique [24] and combine it with the sequential sampling method [17]. This is an important step that allows automating the generation of scalable macromodels and reducing the efforts needed by designers to use scalable macromodels as a design tool. The corresponding main modeling steps are recalled briefly in what follows. The reader can refer to $[15,16,24]$ for more detailed explanations. $N$ dimensional hyper-rectangular ( $N$-box) regions are used as a building block for the design space. The design space is decomposed in a concatenation of several of such regions which are denoted as $\Omega_{l}, l=1, \ldots, L$ related to the estimation data samples.

Each of these $\Omega_{l}$ regions contains $2^{N}$ frequency-dependent rational models called root macromodels at the corresponding corner points. The Laplace variable $s$ is not considered as an element of the design space. It is modeled separately using the root macromodels that are rational pole-residue models of the Laplace variable also known as frequency response functions (FRFs). These root macromodels are identified from the estimation data samples $\left\{\left(s_{n}, \overrightarrow{\boldsymbol{g}}_{\boldsymbol{k}}\right), \boldsymbol{H}\left(s_{n}, \overrightarrow{\boldsymbol{g}}_{\boldsymbol{k}}\right)\right\}$ using the well known Vector Fitting identification technique [25,26]. The root macromodels $\boldsymbol{R}^{\Omega_{l}}\left(s, \overrightarrow{\boldsymbol{g}}_{i}{ }^{\Omega_{l}}\right), i=1, \ldots, 2^{N}$ contained in an $N$-box region $\Omega_{l}$ are represented in a pole-residue form as

$$
\boldsymbol{R}^{\Omega_{l}}\left(s, \overrightarrow{\boldsymbol{g}}_{i}{ }^{\Omega_{l}}\right)=\sum_{p=1}^{P_{i}^{\Omega_{l}}} \frac{\boldsymbol{C}_{p, i}^{\Omega_{l}}}{s-a_{p, i}^{\Omega_{l}}}+\boldsymbol{D}_{i}^{\Omega_{l}}
$$

where $\boldsymbol{C}_{p, i}^{\Omega_{l}}$ represents the residue matrices, $a_{p, i}^{\Omega_{l}}$ denotes the poles and $\boldsymbol{D}_{i}^{\Omega_{l}}$ is the direct-term matrix.

Then, for each $N$-box region $\Omega_{l}$ a set of amplitude and frequency scaling coefficients is computed and an interpolation process of FRFs and scaling coefficients is used to generate a scalable macromodel $\boldsymbol{R}^{\Omega_{l}}(s, \overrightarrow{\boldsymbol{g}})[15,16,24]$. The Mean Absolute Error (MAE) measure or 
the $L 1$-norm per port is used to assess the accuracy of the model in every $N$-box region of the design space at the corresponding validation points

$$
E^{\mathrm{MAE}}(\overrightarrow{\boldsymbol{g}})=\max _{\substack{u=1, \ldots, P \\ v=1, \ldots, P}} \frac{1}{N_{s}}\left(\sum_{n=1}^{N_{s}}\left|R_{u, v}\left(s_{n}, \overrightarrow{\boldsymbol{g}}\right)-H_{u, v}\left(s_{n}, \overrightarrow{\boldsymbol{g}}\right)\right|\right) .
$$

where $H_{u, v}(s, \vec{g})$ and $R_{u, v}(s, \overrightarrow{\boldsymbol{g}})$ denote the EM simulation response and scalable macromodel response, respectively. $P$ is the number of system ports. The MAE error measure or the L1-norm gives a global view on the error between the EM and model frequency responses. We note that an user can decide to utilize another error measure that is more suitable to his modeling needs. If a fixed set of estimation and validation data samples is available, each region $\Omega_{l}$ in the design space is modeled and the corresponding model validated. Cell by cell the complete design space is covered. In the next section, it is briefly described the sequential sampling method [17] that is coupled with the scalable macromodeling method [24] to provide an automated generation of scalable macromodels.

\section{$2.2 \quad$ Automated design space sampling using sequential sampling}

The sequential sampling algorithm [17] is used in this work and it allows automating the generation of scalable macromodels built by the methods [24], while also reducing the computational effort needed to gather estimation and validation data samples. Fig. 1 shows the flowchart of the algorithm and the different steps are discussed below:

1. Initialization: During this step the design space is defined. It contains $N$ design parameters of interest $\overrightarrow{\boldsymbol{g}}=\left(g^{(1)}, \ldots, g^{(N)}\right)$. The initial design space is defined by $2^{N}$ corner points to form one single $N$-box region $\Omega_{l}$ with $l=L=1$.

2. Scalable macromodeling : For each elementary region $\Omega_{l}, l=1, \ldots, L$, a scalable macromodel $\mathbf{R}^{\Omega_{l}}(s, \vec{g})$ is built as explained in Section 2.1 . 
3. Model validation: The selected region $\Omega_{l}$ is validated with respect to the actual EM solver. This requires a set of validation EM simulations that is not used for the model estimation. This is done in two steps: first the EM solver response is compared with the macromodel one using the MAE measure (2) at the center of the maximum sensitive edge (which is the most difficult to be modeled) and if the model is accurate enough, a second level of accuracy check is performed at the geometric center (similarly to [17]).

4. Refinement: If the accuracy of the model in the region $\Omega_{l}$ lies within the desired accuracy threshold $\Delta$, the region $\Omega_{l}$ is not further divided. Otherwise, the region is split into two subregions along the maximum sensitive edge [17]. The accuracy threshold $\Delta$ can be decided based on the design specifications set by the user. For example, if a minimal attenuation in the stop bands of $-30 \mathrm{~dB}$ is required for the optimal design, the scalable macromodel should be able to describe the filter characteristics up to an accuracy of $-30 \mathrm{~dB}$.

Then, after updating the total number of regions $L$ the algorithm is repeated from Step 2 until all regions $\Omega_{l}$ are covered, i.e. $l=L$.

\section{Including the Scalable Macromodel in the Design Process}

The first step in the design process of a microwave filter is the approximation step that consists of determining a polynomial filter function (e.g. Chebyshev polynomial) that fulfills the user-specified specifications. Then, during the realization step a lumped-element circuit consisting of coupled $L C$ resonators is synthesized to realize the polynomial approximation. The lumped-element circuit can be represented by its coupling coefficients and external quality factors [3]. Next the coupling coefficients and external quality factors 


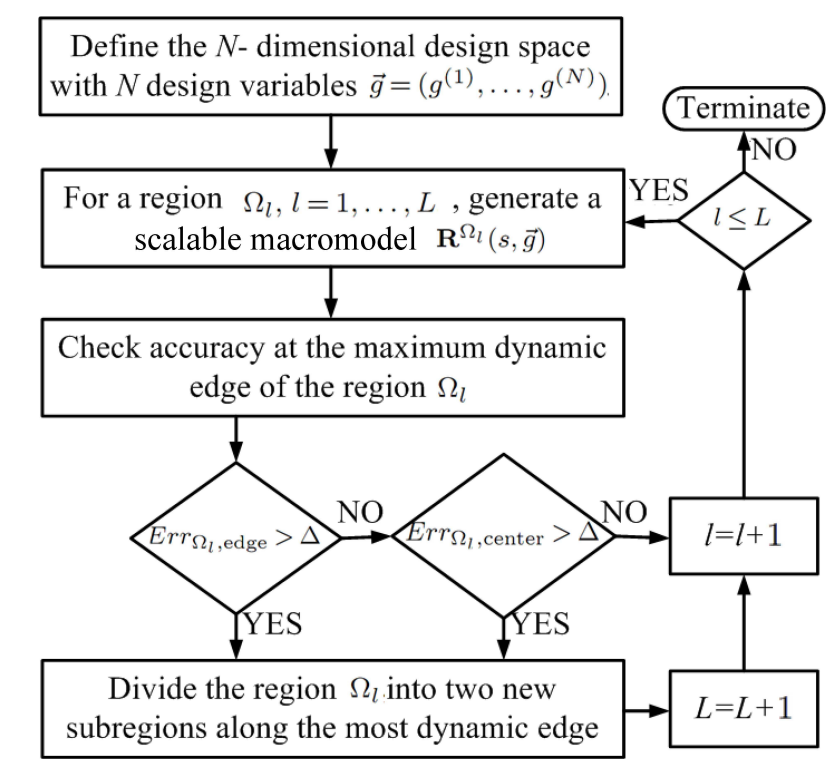

Figure 1: Flowchart of the sequential sampling algorithm.

must be implemented physically in the chosen filter technology. The implementation step consists of physically dimensioning the distributed filter. In general, the implementation step only yields initial physical dimensions and optimization is still required to meet the specifications [2]. Rather than investing time in a brute-force optimization, we propose to invest time in the extraction of a scalable macromodel. Although the model generation process is automated, it still depends on some judicious choices of the user. The frequency range of interest has to be chosen and it is also important to determine for which physical parameters the filter will be modeled, which are called design parameters in this paper. Typically these parameters will be chosen in order to influence properties of the filter response that do not meet the specifications. For example, when the center frequency of the filter is too high, the physical length of the resonators is a parameter that affects this center frequency and thus it can be chosen as a design parameter. Also the ranges of the design parameters must be set. These ranges depend on some practical and/or physical 
considerations. To make this less abstract, let us consider the case of a spacing between two microstrip lines in a coupled line pair. When the spacing is too small, it cannot be realized physically due to fabrication tolerances, while when the spacing becomes too large, there is no more coupling between the lines. Another important choice is the desired accuracy of the macromodel. This choice depends on the given filter specifications. For example, when the required minimal attenuation is equal to $-20 \mathrm{~dB}$, the scalable macromodel should be able to describe the filter characteristics up to an accuracy of $-20 \mathrm{~dB}$. The user may decide to set the model accuracy to a lower number such that there is some margin (e.g. from 5 to $10 \mathrm{~dB}$ of margin). The user choices depend on the design and must thus be made after the initial design step. Once the model is generated it can be used to optimize the initial design. It can however be also used to gain insights about the behavior of the filter with respect to the design parameters or used to optimize for a new set of specifications. How the macromodel is used for optimization purposes is explained in Section 3.1.

\subsection{Optimization}

The scalable macromodel $\boldsymbol{R}(s, \vec{g})$ of a microwave filter can be used to optimize the initial design such that it fulfills the desired specifications. The global optimization function MultiStart in Matlab R2012a [27] is used in this paper to perform global optimization of the filter to satisfy the desired performances. The MultiStart routine first generates uniformly distributed starting points in the design space from which several local optimizer runs are performed, generating multiple solutions. This routine then ranks the solutions in terms of their cost function values in ascending order. It is important to highlight that a global optimization usually requires a high number of function evaluations (and then simulations of the system behavior). This is not computationally expensive if scalable macromodels are used for it. Global optimization is interesting since it searches for design solutions over 
the complete design space of interest.

Considering microwave filters, a typical optimization process begins by defining passband and stopband specifications in terms of the scattering parameter responses (S-parameters), which are reformulated in the form of a cost function $F\left(s_{m}, \overrightarrow{\boldsymbol{g}}\right)$ at optimization frequency samples $s_{m}, m=1,2, . . N_{s}^{\mathrm{OP}}$ to be minimized as a function of the design parameters $\vec{g}$

$$
F\left(s_{m}, \overrightarrow{\boldsymbol{g}}\right)=R_{\mathrm{L}}^{m}-R\left(s_{m}, \overrightarrow{\boldsymbol{g}}\right) \text { or } R\left(s_{m}, \overrightarrow{\boldsymbol{g}}\right)-R_{\mathrm{U}}^{m}
$$

In (3), $R_{\mathrm{L}}^{m}$ and $R_{\mathrm{U}}^{m}$ represents the lower and upper frequency response thresholds, respectively, at frequency samples $s_{m}, m=1, \ldots, N_{s}^{\mathrm{OP}}$, spread over the frequency range of interest. The optimization frequency samples $s_{m}, m=1, \ldots, N_{s}^{\mathrm{OP}}$ can be different from the modeling frequency samples $s_{n}, n=1, \ldots, N_{s}$ described in Section 2.1. The frequency samples $s_{n}$ are generated by the EM solver that is computationally expensive, while the optimization frequency samples $s_{m}$ are generated using the scalable macromodel $\boldsymbol{R}(s, \overrightarrow{\boldsymbol{g}})$ that is efficient to evaluate. This means that, for optimization purposes, more frequency samples could be selected to have a better estimation of the cost function $F\left(s_{m}, \overrightarrow{\boldsymbol{g}}\right)$ (i.e., $N_{s}^{\mathrm{OP}}>N_{s}$ ). A negative value in (3) indicates that the corresponding specification is satisfied, while a positive value denotes that the specification is violated. The final cost function to be optimized is then given as the worst-case violation over all the S-parameters matrix entries and the $s_{m}$ samples

$$
F(\overrightarrow{\boldsymbol{g}})=\max _{i, j} \max _{s_{m}} F_{i, j}\left(s_{m}, \overrightarrow{\boldsymbol{g}}\right) .
$$

where $F_{i, j}$ represents the cost function for the $(i, j)-t h$ S-parameter matrix entry. The cost function (4) is then supplied to the MultiStart optimization routine, resulting in multiple optimal design space points that satisfy the specifications. Such an optimization process 
Table 1: Specifications of the Dual-Band Filter

\begin{tabular}{|c|c|c|}
\hline & $f_{1}$ & $f_{2}$ \\
\hline Center frequency & $2 \mathrm{GHz}$ & $2.65 \mathrm{GHz}$ \\
\hline Bandwidth & $50 \mathrm{MHz}$ & $50 \mathrm{MHz}$ \\
\hline In-band insertion loss & $\leq-3 \mathrm{~dB}$ & $\leq-3 \mathrm{~dB}$ \\
\hline In-band return loss & $\geq-10 \mathrm{~dB}$ & $\geq-10 \mathrm{~dB}$ \\
\hline
\end{tabular}

applied to a filter case will be illustrated in Section 4 .

\section{Example: Microstrip Dual-Band Bandpass Filter}

We have chosen a state-of-the-art microstrip dual-band bandpass filter introduced in [23] to illustrate and validate the proposed approach. In [23], a design method is presented to adjust the center frequency, bandwidth, the position of the transmission zeros and desired ratio of the resonance frequency of the two passbands. The filter consists of two coupled unequal length shunted-line stepped impedance resonators (see Fig. 2). We use the design method presented in [23] to obtain initial values of the design parameters for the initial design. The filter is fabricated on a RO4003 substrate with a relative permittivity $\epsilon_{r}$ equal to 3.55 , a dielectric height of $1.542 \mathrm{~mm}$ and a loss tangent $\delta$ equal to 0.0022 . The EM solver used to generate data samples of the filter response is ADS2011 Momentum [28]. All numerical experiments were performed using Matlab R2012A [27] and on Windows platform equipped with Intel Core2 Extreme CPU Q9300 $2.53 \mathrm{GHz}$ and 8 GB RAM. First, the steps to obtain the initial design are discussed in what follows.

\subsection{Approximation Step}

The specifications of the filter are summarized in Table 1. The design method in [23] proposes to approximate each band separately with a Chebyshev response of order 2 . 


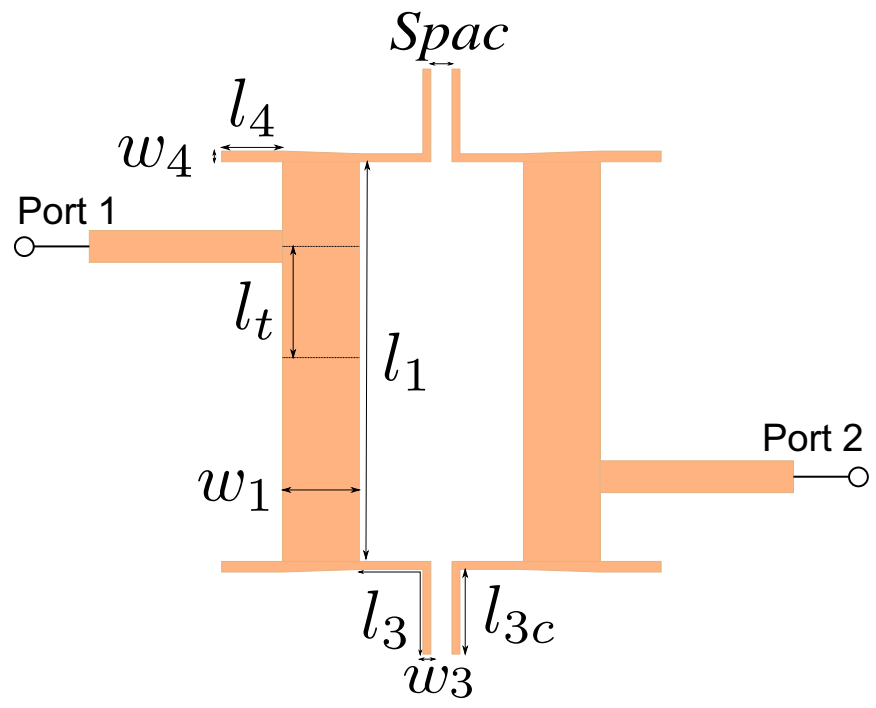

Figure 2: Topview of the layout of the filter.

Table 2: Coupling Coefficients and External Quality Factors Obtained during the Realization Step

\begin{tabular}{|c|c|c|}
\hline & $f_{1}$ & $f_{2}$ \\
\hline$M_{1,2}$ & 0.0345 & 0.026 \\
\hline$Q_{e i}$ & 33.7 & 44.8 \\
\hline$Q_{e o}$ & 33.7 & 44.8 \\
\hline
\end{tabular}

\section{$4.2 \quad$ Realization Step}

In the case of a Chebyshev response, formulas exist to determine the low-pass prototype parameters $g_{i}$ of the equivalent lumped circuit [29]. The corresponding coupling coefficients and external quality factors can be determined as follows:

$$
\begin{gathered}
Q_{e i}=\frac{g_{0} g_{1}}{F B W} \\
Q_{e o}=\frac{g_{n} g_{n+1}}{F B W}
\end{gathered}
$$




$$
M_{i, i+1}=\frac{F B W}{\sqrt{g_{i} g_{i+1}}}, \text { for } i=1 \text { to } n-1
$$

where $Q_{e i}$ and $Q_{e o}$ are the input and output external quality factors, respectively and $M_{i, i+1}$ is the coupling coefficient between the adjacent resonators. $F B W$ is the fractional bandwidth of the passband. $n$ is the order of the filter, which is equal to 2 in this case. Applying equations (5)-(7) yields the results summarized in Table 2.

\subsection{Implementation Step: Initial Design}

The initial values for the design parameters are obtained by applying the procedure described in [23]. In this section, we briefly summarize this procedure focusing on the effects that the parameters have on the center frequency of the bands, the coupling coefficients and the external quality factors.

The lengths $l_{1}, l_{3}, l_{3 c}$ and $l_{4}$ are chosen such that resonator is a half-wavelength resonator

that resonates at $f_{1}$. The impedance ratios $\frac{Z_{01}}{Z_{03}}$ and $\frac{Z_{01}}{Z_{04}}$ determine the ratio $\frac{f_{2}}{f_{1}}$. Decreasing the values of $\frac{Z_{01}}{Z_{03}}$ and $\frac{Z_{01}}{Z_{04}}$ leads to decrease the value of $\frac{f_{2}}{f_{1}}$. The widths $w_{1}, w_{3}$ and $w_{4}$ are the physical parameters that mainly determine $Z_{01}, Z_{03}$ and $Z_{04}$ respectively. The values of $w_{1}, w_{3}$ and $w_{4}$ are chosen such that $\frac{f_{2}}{f_{1}}=1.325$.

The tapping position $l_{t}$ and the lengths $l_{3 c}$ and $l_{4}$ mainly affect $Q_{e i}$ and $Q_{e o}$. They are chosen such that the physical external quality factors are approximately the same as the ones found during the realization step. Initial values for these parameters are however difficult to determine, since they affect both the bands. Therefore, these values of the design parameters are chosen such that both physical $Q_{e i}$ and $Q_{e o}$ are acceptably close to the theoretical ones. The physical $Q_{e i}$ and $Q_{e o}$ are shown in Table 3.

The spacing parameter Spac between the resonators mainly affects the coupling coefficient $M_{12} . w_{3}$ and $w_{4}$ also affect the coupling coefficient, but their effect is smaller than 
Table 3: Coupling Coefficients and External Quality Factors (EM simulations)

\begin{tabular}{|c|c|c|}
\hline & $f_{1}$ & $f_{2}$ \\
\hline$M_{1,2}$ & 0.0373 & 0.0327 \\
\hline$Q_{e i}$ & 25 & 33.5 \\
\hline$Q_{e o}$ & 25 & 33.5 \\
\hline
\end{tabular}

Table 4: Initial Values of the Design Parameters

\begin{tabular}{|c|c|}
\hline$l_{1}$ & $24.5 \mathrm{~mm}$ \\
\hline$l_{3}$ & $17 \mathrm{~mm}$ \\
\hline$l_{3 c}$ & $10 \mathrm{~mm}$ \\
\hline$l_{4}$ & $1.5 \mathrm{~mm}$ \\
\hline$l_{t}$ & $8.8 \mathrm{~mm}$ \\
\hline$w_{1}$ & $9.5 \mathrm{~mm}$ \\
\hline$w_{3}$ & $1 \mathrm{~mm}$ \\
\hline$w_{4}$ & $1 \mathrm{~mm}$ \\
\hline Spac & $1.62 \mathrm{~mm}$ \\
\hline
\end{tabular}

that one due to Spac. The values of Spac, $w_{3}$ and $w_{4}$ are chosen such that the physical coupling coefficient $M_{12}$ is approximately that one found during the realization step. The physical $M_{12}$ for both bands is shown in Table 3 .

The initial values of the design parameters given by this design method are summarized in Table 4. Figs. 3 -4 show the magnitude of $S_{21}$ and $S_{11}$ for the initial design, respectively. It is clear that specifications are not met and therefore optimization is needed.

\subsection{Design Space}

In this section, we describe how we select which physical parameters are chosen to become design parameters. Fig. 3 shows that the ratio $\frac{f_{2}}{f_{1}}$ is not equal to 1.325 , but that $f_{1}=2$ GHz. Since the ratio $\frac{f_{2}}{f_{1}}$ is mainly affected by $\frac{Z_{01}}{Z_{03}}$ and $\frac{Z_{01}}{Z_{04}}, w_{1}$ and $w_{3}=w_{4}$ are chosen as design parameters. The ranges are determined as follows: $w_{1}$ must not become too large, because for large values of $w_{1}$ the characteristic impedance $Z_{01}$ does not decrease anymore, so it is not relevant to model the filter behavior for those values. The value of $w_{1}$ must 


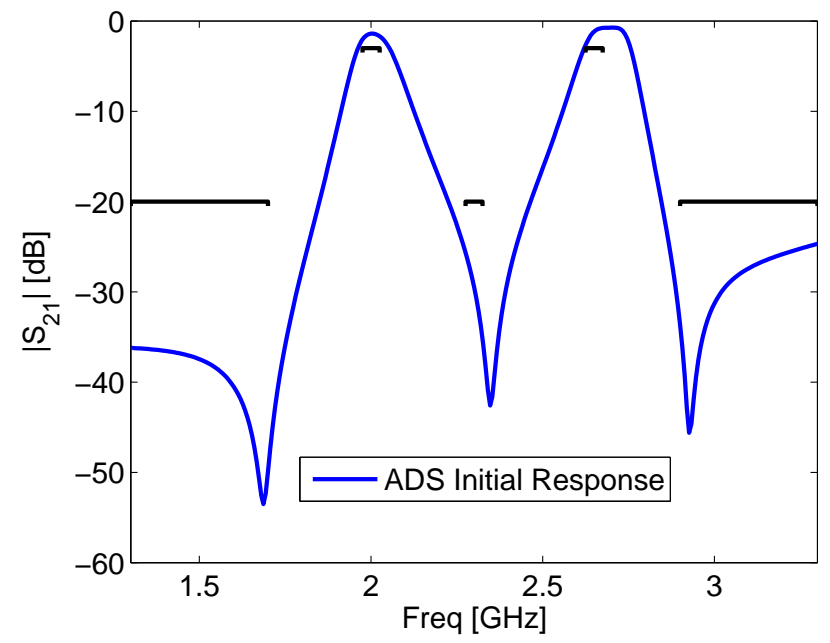

Figure 3: Magnitude of $S_{21}$ for the initial design (blue) and the specifications (black).

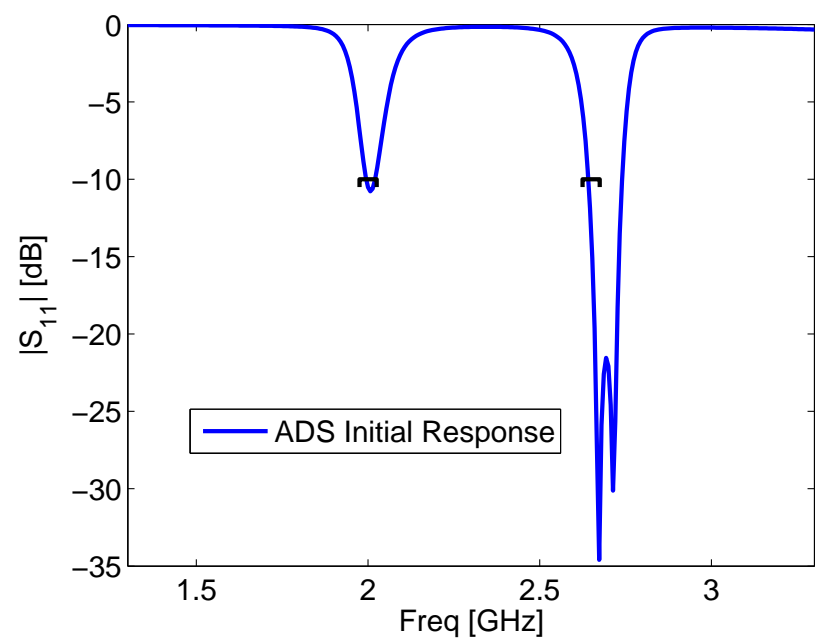

Figure 4: Magnitude of $S_{11}$ for the initial design (blue) and the specifications (black).

not become too small either, because then the ratio $\frac{Z_{01}}{Z_{03}}$ becomes too large and $\frac{f_{2}}{f_{1}}$ becomes too large. The value of $w_{3}$ must not become too small, because then it is not physically realizable. It must also not become too large, because then the ratio $\frac{Z_{01}}{Z_{03}}$ becomes too large and $\frac{f_{2}}{f_{1}}$ becomes too large. Since the found coupling coefficient between the resonators is 
Table 5: Ranges of the design parameters.

\begin{tabular}{|c|c|}
\hline Parameter & Range \\
\hline \hline$w_{1}$ & $8.5-10.5 \mathrm{~mm}$ \\
\hline$w_{3}$ & $0.5-2 \mathrm{~mm}$ \\
\hline Spac & $0.5-2 \mathrm{~mm}$ \\
\hline
\end{tabular}

too large, Spac is also chosen as a design parameter. The upper bound of the range of Spac is chosen to make sure that there is still electromagnetic coupling between the resonators and the lower bound is chosen such that it is physically realizable. Table 5 summarizes the ranges of the design parameters.

\subsection{Generation of the Scalable Macromodel}

Sections 2.1 and 2.2 show that the generation of scalable macromodels is automated. Nevertheless, the user has to specify the design space, the frequency span and the accuracy for the model. The choice of the design space $\overrightarrow{\boldsymbol{g}}=\left[w_{1}, w_{2}\right.$, Spac $]$ has been previously discussed. The frequency span is chosen equal to freq $\in[1.3-3.3] \mathrm{GHz}$ to be wide enough such that the filter behavior of interest is within it. The choice of the model accuracy is based on the minimal attenuation desired in the stopband that is equal to $-30 \mathrm{~dB}$ in this numerical example. The accuracy of the scalable macromodel has been set to $-30 \mathrm{~dB}$ considering the MAE measure (2).

The scalable macromodeling method [24] along with the sequential sampling scheme discussed in Section 2 have been implemented in Matlab R2012a and used to drive the ADS2011 Momentum software with Adaptive Frequency Sampling (AFS) [28] to generate the S-parameters data samples at selected design space samples. AFS is a technique included in ADS2011 Momentum that adaptively samples the frequency range and can be used to then efficiently provide the system response over a specified number of frequency points. The number of simulated frequency samples $N_{s}$ obtained by using AFS over the 
Table 6: Scalable macromodeling

\begin{tabular}{|c|c|c|c|c|}
\hline \multicolumn{2}{|c|}{ \# Samples } & CPU Time & Accuracy \\
\hline Generation & Validation & Modeling & Data Gen. & {$[\mathrm{dB}]$} \\
\hline 72 & 45 & 36 min $35 \mathrm{~s}$ & $1 \mathrm{~h} 55 \mathrm{~min} 21 \mathrm{~s}$ & -30.48 \\
\hline Average CPU Time for one ADS frequency sweep $=50 \mathrm{~s}$ \\
Average CPU Time for one macromodel frequency sweep $=19.5 \mathrm{~ms}$ \\
Speed-up = $2564 \times$ \\
\hline
\end{tabular}

range freq $\in[1.3-3.3] \mathrm{GHz}$ has been chosen equal to 301 to build the scalable macromodel, so that the sharp behavior of the S-response of the microwave filter with respect to frequency is well captured.

The MAE measure (2) is used to asses the accuracy of the scalable macromodel. Table 6 reports the total number of design space samples (estimation and validation), the worst case MAE (2) over the estimation and validation data, the CPU time needed to run all the ADS Momentum estimation and validation simulations and the CPU time needed to obtain the scalable macromodel using the sequential scheme coupled with the scalable macromodeling method [24]. The average CPU time needed by ADS Momentum (using AFS) and the scalable macromodel for one frequency sweep over 301 frequency points is also shown in Table 6. This measure is crucial to judge the advantage of the proposed approach for efficient design optimizations instead of using EM-based optimization schemes.

\subsection{Filter optimization}

Once the scalable macromodel has been generated, it is used to perform multiple optimizations for this filter. The optimization specifications on the S-parameters of the filter under study are: 


$$
\begin{array}{ll}
\left|S_{21}\right|<-L_{A 1} \mathrm{~dB} & f_{s 1} \leq \text { freq } \leq f_{s 2}, \\
\left|S_{21}\right|>-L_{I L} \mathrm{~dB} & f_{p 1} \leq \text { freq } \leq f_{p 2}, \\
\left|S_{11}\right|<-L_{R L} \mathrm{~dB} & f_{p 1} \leq \text { freq } \leq f_{p 2}, \\
\left|S_{21}\right|<-L_{A 2} \mathrm{~dB} & f_{s 3} \leq \text { freq } \leq f_{s 4}, \\
\left|S_{21}\right|>-L_{I L} \mathrm{~dB} & f_{p 3} \leq \text { freq } \leq f_{p 4}, \\
\left|S_{11}\right|<-L_{R L} \mathrm{~dB} & f_{p 3} \leq \text { freq } \leq f_{p 4}, \\
\left|S_{21}\right|<-L_{A 3} \mathrm{~dB} & f_{s 5} \leq \text { freq } \leq f_{s 6} .
\end{array}
$$

with three optimization cases:

$I .\left(f_{s 1}, f_{s 2}, f_{p 1}, f_{p 2}, f_{s 3}, f_{s 4}, f_{p 3}, f_{p 4}, f_{s 5}, f_{s 6}\right)=$

$(1.3,1.7,1.975,2.025,2.275,2.325,2.625,2.675,2.9,3.3) \mathrm{GHz}$,

$\left(L_{A 1}, L_{I L}, L_{R L}, L_{A 2}, L_{A 3}\right)=(-20,-3,-10,-20,-20) \mathrm{dB}$

II. $\left(f_{s 1}, f_{s 2}, f_{p 1}, f_{p 2}, f_{s 3}, f_{s 4}, f_{p 3}, f_{p 4}, f_{s 5}, f_{s 6}\right)=$

$(1.3,1.7,1.975,2.025,2.275,2.325,2.625,2.675,2.9,3.3) \mathrm{GHz}$,

$\left(L_{A 1}, L_{I L}, L_{R L}, L_{A 2}, L_{A 3}\right)=(-20,-3,-10,-30,-20) \mathrm{dB}$

III. $\left(f_{s 1}, f_{s 2}, f_{p 1}, f_{p 2}, f_{s 3}, f_{s 4}, f_{p 3}, f_{p 4}, f_{s 5}, f_{s 6}\right)=$

$(1.3,1.6,1.9,1.95,2.175,2.25,2.625,2.675,2.9,3.3) \mathrm{GHz}$,

$\left(L_{A 1}, L_{I L}, L_{R L}, L_{A 2}, L_{A 3}\right)=(-20,-3,-10,-20,-20) \mathrm{dB}$ 
Table 7: Dual-band bandpass filter: global optimization results.

\begin{tabular}{|c|c|c|c|c|}
\hline Case & $\begin{array}{c}\text { Initial design } \\
\left(w_{1}, w_{2}, \text { Spac }\right)[\mathrm{mm}]\end{array}$ & $\begin{array}{c}\text { Best optimal design } \\
\left(w_{1}^{*}, w_{2}^{*}, S p a c^{*}\right)[\mathrm{mm}]\end{array}$ & $\begin{array}{c}\text { \# Function } \\
\text { evaluations }\end{array}$ & $\begin{array}{c}\text { Optimization time } \\
\text { using the macromodel [s] }\end{array}$ \\
\hline I & {$[9.5,1,1.62]$} & {$[9.66,1,1.44]$} & 6557 & $5 \mathrm{~min}, 40 \mathrm{~s}$ \\
\hline II & {$[9.5,1,1.62]$} & {$[10.4,1.17,1.38]$} & 5456 & $5 \mathrm{~min}, 47 \mathrm{~s}$ \\
\hline III & {$[9.5,1,1.62]$} & {$[10.3,1.43,1.25]$} & 6675 & $5 \mathrm{~min}, 43 \mathrm{~s}$ \\
\hline
\end{tabular}

As explained in Section 3.1, the global optimization function MultiStart in Matlab R2012a is used to perform global optimization with a cost function (4) using the specifications previously described and considering 30 starting points for each optimization case. For each of the three optimization cases, the function MultiStart has found multiple optimization solutions $\left[w_{1}^{*}, w_{3}^{*}, S p a c^{*}\right]$ that satisfy the corresponding specifications. The results of the three optimization cases are tabulated in Table 7, where the best optimization solutions, the total number of function evaluations and CPU time needed for the three global optimizations are shown.

Considering the average CPU time needed for one frequency sweep using the EM solver and the scalable macromodel (see Table 6) and the number of functions evaluations needed to perform the global optimizations in Table 7 , it is clear the using the scalable macromodel definitely allows very efficient multiple global optimization steps. The initial computational effort needed to generate the scalable macromodel (see Table 6) becomes a little effort when the CPU time saved to perform the multiple global optimizations is considered.

Figs. 5-6 show the optimization results for Case I and the different S-parameters before and after optimization. The specifications are shown by the solid black lines and as evident from the two figures, all the specifications are satisfied. Similar curves are observed for 


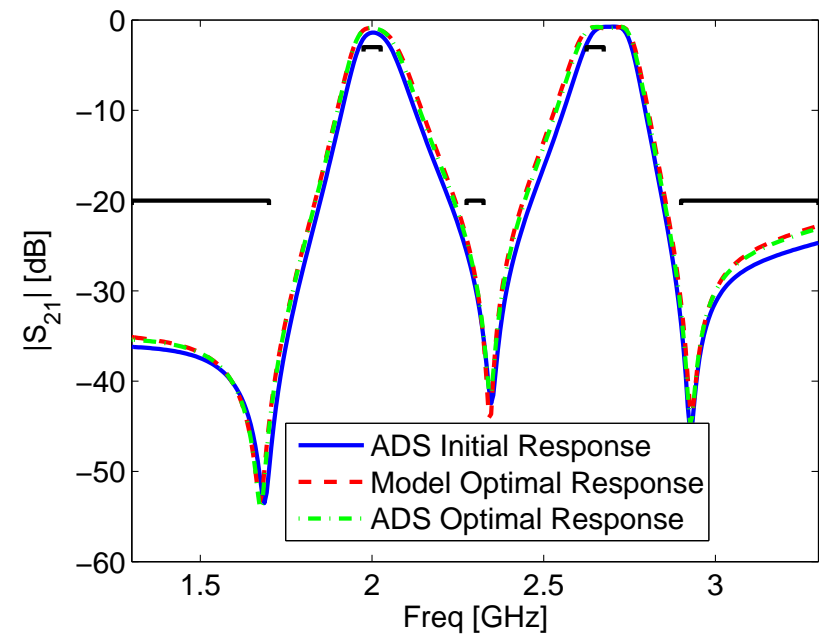

Figure 5: Case I: Magnitude of $S_{21}$ before and after optimization.

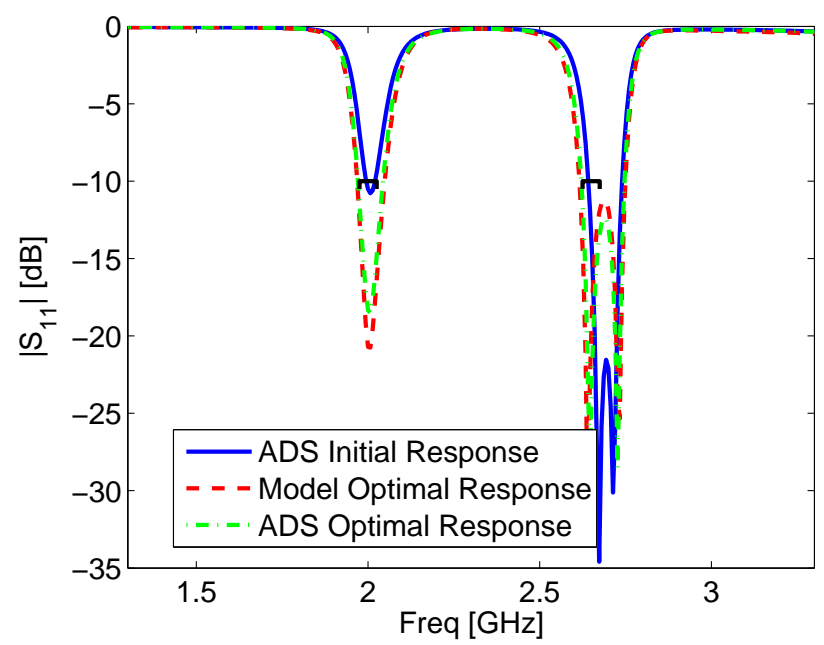

Figure 6: Case I: Magnitude of $S_{11}$ before and after optimization.

Case II and Case III as shown in Figs. 7-10. The ADS optimal response curves denote the ADS EM simulations performed at the optimal solution points to verify that the model prediction is accurate. 


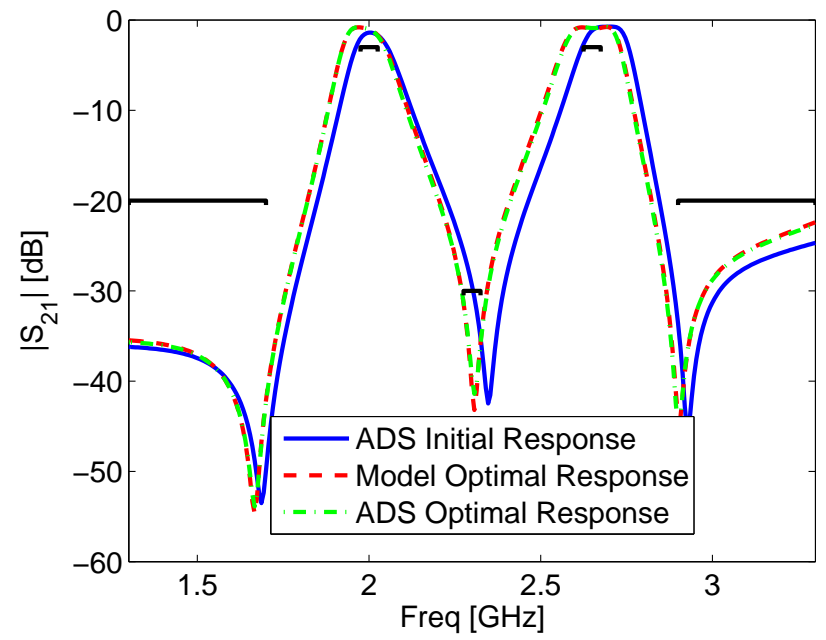

Figure 7: Case II: Magnitude of $S_{21}$ before and after optimization.

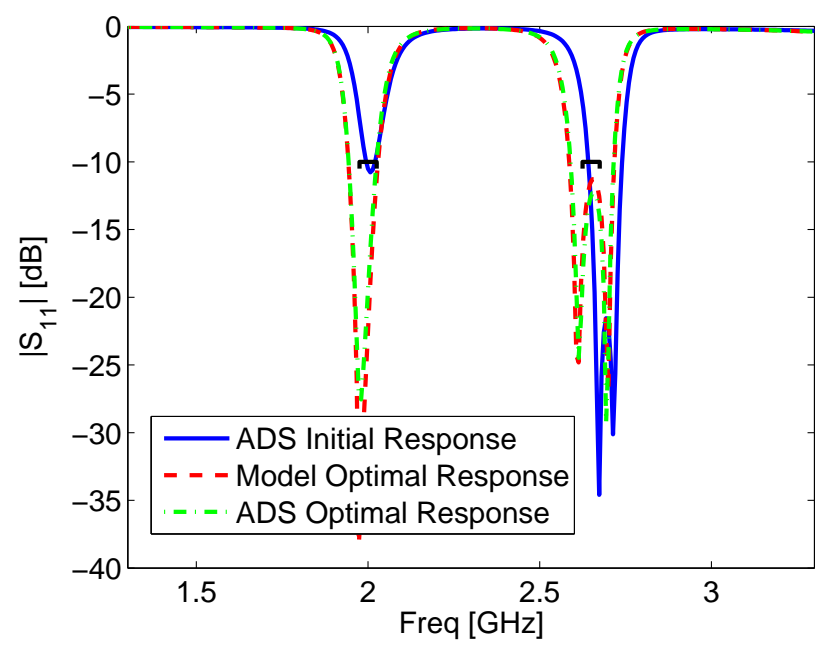

Figure 8: Case II: Magnitude of $S_{11}$ before and after optimization.

\section{Discussion}

Generating scalable macromodels becomes more computationally expensive with an increasing number of design parameters. The so called "curse of dimensionality" pops up in high-dimensional modeling problems. This affects two main aspects of the modeling: 1) 


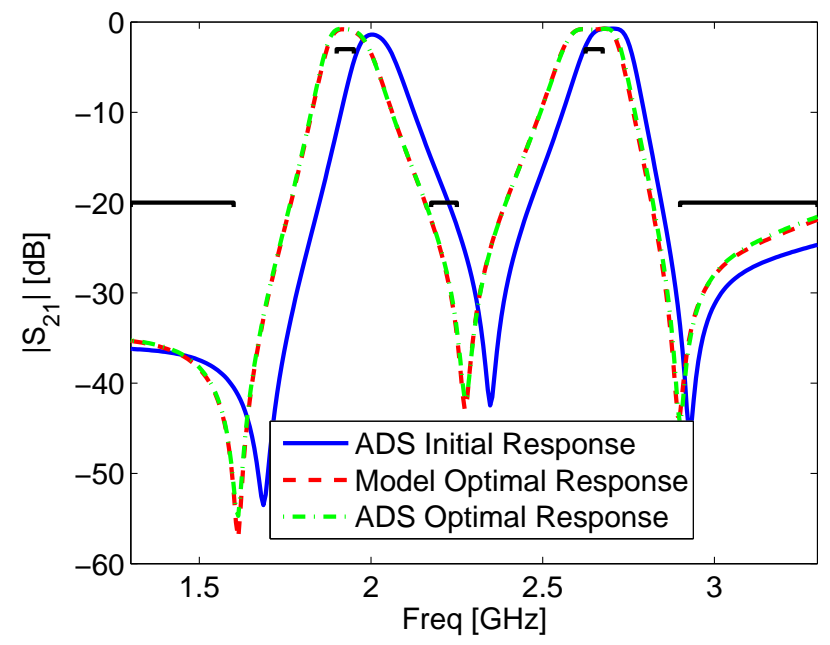

Figure 9: Case III: Magnitude of $S_{21}$ before and after optimization.

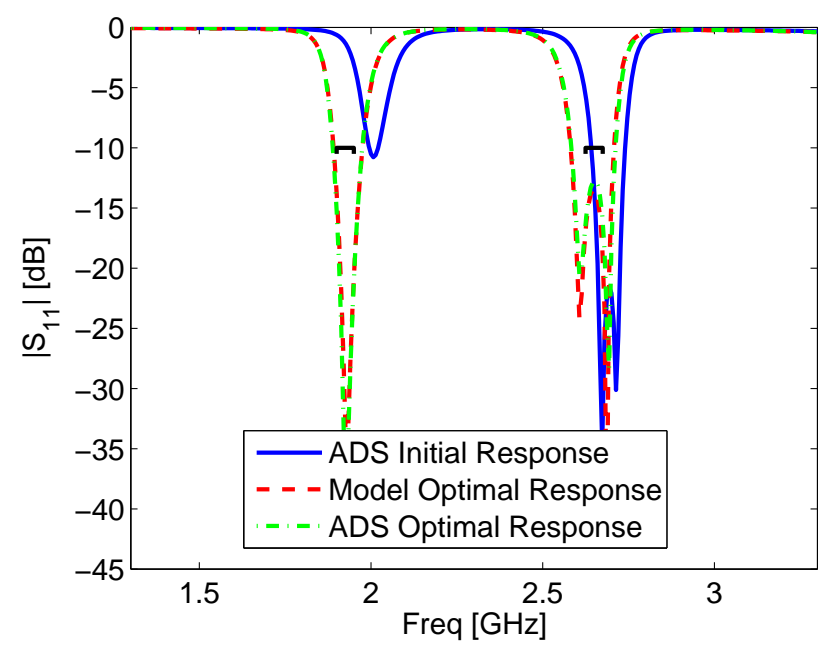

Figure 10: Case III: Magnitude of $S_{11}$ before and after optimization.

the number of data samples (and then the CPU time to collect these data samples) needed to build and validate a model and 2) the complexity (and then the CPU time) of the model generation when the data samples are available.

Concerning the first aspect, working on fully regular design space grids to collect estima- 
tion and validation data to then build and validate a scalable macromodel will make the complexity of the data gathering increase in an exponential way with respect to the number of design parameter. This issue can be mitigated by using sequential sampling strategies that optimizes the samples location in the design space.

Concerning the second aspect, the modeling step for the technique [24] whose complexity will mainly suffer from an increasing number of design parameters is the computation of the amplitude and frequency scaling coefficients for each region of the design space obtained during the sequential sampling strategy. This issue can be mitigated by exploiting parallelization strategies, since the scaling coefficients computation for a design space region can be performed independently from the other regions.

As future work, we plan to investigate high-dimensional scalable macromodeling approaches and strategies to deal with the curse of dimensionality.

\section{Conclusion}

In this paper, we have introduced scalable macromodels in the design cycle of microwave filters. We have discussed how to generate and use scalable macromodels as a design tool for filters. The main advantage of these macromodels is that they are cheap to evaluate with a suitable accuracy. Hence, the scalable macromodels can replace the expensive EM solver in multiple optimizations of the filter and then make these steps much less CPU time consuming. It is to be noted that it also takes an initial computational effort to generate the macromodel and that this must be done during the design cycle. However, this initial computational effort needed to generate the scalable macromodel becomes a little effort when the CPU time saved to perform multiple global optimizations with respect to EMbased optimizations is considered. We have illustrated this macromodeling-based design approach by applying it to the design of a state-of-the-art microstrip dual-band bandpass 
filter. Although the generation of the scalable macromodels is an automated process, it still requires some information from a designer, namely the frequency span, the ranges of the design parameters and the desired model accuracy. How a designer can hand this information to the macromodel generation process has been explained in detail. We have finally show how the scalable macromodel can be used for multiple optimizations. The corresponding numerical results confirm that the macromodeling-based design approach works very well.

\section{References}

[1] Levy, R., Snyder, R.V., Matthaei, G.: 'Design of microwave filters', IEEE Transactions on Microwave Theory and Techniques, 2002, 50, (3), pp. 783-793

[2] Swanson, D., Macchiarella, G.: 'Microwave filter design by synthesis and optimization', IEEE Microwave Magazine, 2007, 8, (2), pp. 55-69

[3] Hong, J.-S. G., Lancaster, M. J.: 'Microstrip filters for RF/microwave applications' (Wiley-interscience, 2001, 1st edn., 2001)

[4] Peik, S., Mansour, R., Chow, Y.: 'Multidimensional Cauchy method and adaptive sampling for an accurate microwave circuit modeling', IEEE Transactions on Microwave Theory and Techniques, 1998, 46, (12), pp. 2364-2371

[5] Lamecki,A., Kozakowski, P., Mrozowski, M.: 'Efficient implementation of the Cauchy method for automated CAD-model construction', IEEE Microwave and Wireless Components Letters, 2003, 13, (7), pp. 268-270 
[6] Cuyt, A., Lenin, R., Becuwe, S., et al.: 'Adaptive multivariate rational data fitting with applications in electromagnetics': IEEE Transactions on Microwave Theory and Techniques, 2006, 54, (5), pp. 2265-2274

[7] Devabhaktuni, V., Chattaraj, B., Yagoub, M., et al.: 'Advanced microwave modeling framework exploiting automatic model generation, knowledge neural networks, and space mapping', IEEE Transactions on Microwave Theory and Techniques, 2003, 51, (7), pp. $1822-1833$

[8] Lamecki, A., Balewski, L., Mrozowski, M.: 'Adaptive CAD-model construction schemes', IEEE Transactions on Magnetics, 2009, 45, (3), pp. 1538-1541

[9] Basl, P., Gohary, R., Bakr, M., et al. 'Modelling of electromagnetic responses using a robust multi-dimensional Cauchy interpolation technique', IET Microwaves, Antennas Propagation, 2010, 4, (11), pp. 1955-1964

[10] Lehmensiek, R., Meyer, P.: 'Creating accurate multivariate rational interpolation models of microwave circuits by using efficient adaptive sampling to minimize the number of computational electromagnetic analyses', IEEE Transactions on Microwave Theory and Techniques, 2001, 49, (8), pp. 1419-1430

[11] Triverio, P., Grivet-Talocia, S., Nakhla, M.: 'A parameterized macromodeling strategy with uniform stability test', IEEE Transactions on Advanced Packaging, 2009, 32, (1), pp. 205-215

[12] Ferranti, F., Knockaert, L., Dhaene, T.: 'Parameterized S-parameter based macromodeling with guaranteed passivity', IEEE Microwave and Wireless Component Letters, 2009, 19, (10), pp. 608-610 
[13] Ferranti, F., Knockaert, L., Dhaene, T.: 'Guaranteed passive parameterized admittance-based macromodeling', IEEE Transactions on Advanced Packaging, 2010, 33, (3), pp. 623-629

[14] Triverio, P., Nakhla, M., Grivet-Talocia, S.: 'Passive parametric macromodeling from sampled frequency data', IEEE International Conference on Signal Propagation and Interconnects, Hildesheim, Germany, May 2010, pp. 117-120

[15] Ferranti, F., Knockaert, L., Dhaene, T.: 'Passivity-preserving parametric macromodeling by means of scaled and shifted state-space systems', IEEE Transactions on Microwave Theory and Techniques, 2011, 59, (10), pp. 2394-2403

[16] Ferranti, F., Knockaert, L., Dhaene, T., et al.: 'Parametric macromodeling based on amplitude and frequency scaled systems with guaranteed passivity', International Journal of Numerical Modelling: Electronic Networks, Devices and Fields, 2012, 25, (2), pp. 139-151

[17] Chemmangat, K., Ferranti, F., Dhaene, T., et al.: 'Scalable models of microwave system responses using sequential sampling on unstructured grids', Int. J. of Numerical Modelling: Electronic Networks, Devices and Fields, 2014, 27, (1), pp. 122137

[18] Koziel, S., Bandler, J., Madsen, K.: 'A space-mapping framework for engineering optimization; theory and implementation', IEEE Transactions on Microwave Theory and Techniques, 2006, 54, (10), pp. 3721-3730

[19] Koziel, S, Bandler, J., Cheng, Q.: 'Robust trust-region space-mapping algorithms for microwave design optimization', IEEE Transactions on Microwave Theory and Techniques, 2010, 8, (8), pp. 2166-2174 
[20] Koziel, S: 'Shape-preserving response prediction for microwave design optimization', IEEE Transactions on Microwave Theory and Techniques, 2010 58, (11), pp. 2829-2837

[21] Couckuyt, I., Declercq, F., Dhaene, T., et al.: 'Surrogate-based infill optimization applied to electromagnetic problems', International Journal of RF and Microwave Computer-Aided Engineering, 2010, 20, (5), pp. 492-501

[22] Couckuyt, I., Declercq, F., Dhaene, T.: 'Towards efficient multiobjective optimization: Multiobjective statistical criterions', Brisbane, Australia, June 2012, pp. 1-8.

[23] Hsu, C.-Y., Chen, C.-Y., Chuang, H.-R.: 'Microstrip dual-band bandpass filter design with closely specified passbands', IEEE Transactions on Microwave Theory and Techniques, 2013, 61, (1), pp. 98-106

[24] Chemmangat, K., Ferranti, F., Dhaene, T., et al.: 'Parametric macromodelling of linear high-frequency systems using multiple frequency scaling and sequential sampling', Electronics Letters, 2014, 50, (6), pp. 475-476

[25] Gustavsen, B., Semlyen, A.: 'Rational approximation of frequency domain responses by vector fitting', IEEE Transactions on Power Delivery, 1999, 14, (3), pp. 1052-1061, 1999

[26] Gustavsen, B.: 'Improving the pole relocating properties of vector fitting', IEEE Transactions on Power Delivery, 2006, 21, (3), pp. 1587-1592,

[27] MATLAB, R2012a. Natick, Massachusetts: The MathWorks Inc., 2012

[28] Agilent Technologies, 'Advanced design system', 2011, santa Rosa, CA.

[29] Matthaei, G.L., Young, L., Jones, E.: 'Microwave filters, impedance-matching networks, and coupling structures'(McGraw-Hill New York, 1963, 5th edn., 1964) 